ARTICLE

\title{
Manipulation of current rectification in van der Waals ferroionic CulnP ${ }_{2} \mathrm{~S}_{6}$
}

Xingan Jiang ${ }^{1}$, Xueyun Wang $\mathbb{0}^{1 凶}$, Xiaolei Wang ${ }^{2 凶}{ }^{凶}$, Xiangping Zhang ${ }^{1}$, Ruirui Niu ${ }^{3}$, Jianming Deng ${ }^{1}$

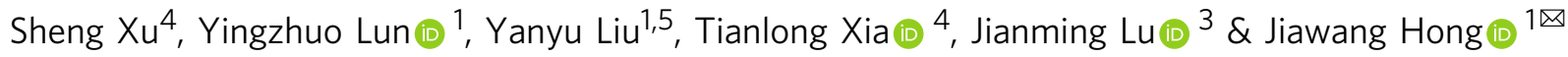

Developing a single-phase self-rectifying memristor with the continuously tunable feature is structurally desirable and functionally adaptive to dynamic environmental stimuli variations, which is the pursuit of further smart memristors and neuromorphic computing. Herein, we report a van der Waals ferroelectric CulnP $\mathrm{S}_{6}$ as a single memristor with superior continuous modulation of current and self-rectifying to different bias stimuli (sweeping speed, direction, amplitude, etc.) and external mechanical load. The synergetic contribution of controllable $\mathrm{Cu}$ + ions migration and interfacial Schottky barrier is proposed to dynamically control the current flow and device performance. These outstanding sensitive features make this material possible for being superior candidate for future smart memristors with bidirectional operation mode and strong recognition to input faults and variations.

\footnotetext{
${ }^{1}$ School of Aerospace Engineering, Beijing Institute of Technology, 100081 Beijing, China. ${ }^{2}$ College of Physics and Optoelectronics, Faculty of Science, Beijing University of Technology, 100124 Beijing, China. ${ }^{3}$ State Key Laboratory for Mesoscopic Physics and Frontiers Science Center for Nano-optoelectronics, School of Physics, Peking University, 100871 Beijing, China. ${ }^{4}$ Department of Physics and Beijing Key Laboratory of Opto-electronic Functional Materials \& Micro-nano Devices, Renmin University of China, 100871 Beijing, China. ${ }^{5}$ College of Physics and Materials Science, Tianjin Normal University, 300387

Tianjin, PR China. 凶email: xueyun@bit.edu.cn; xiaoleiwang@bjut.edu.cn; hongjw@bit.edu.cn
} 
ecent advances in controlling the ionic migration processes in solid-state thin films have led to the rapid development of ionic functionalities ${ }^{1,2}$. One representative application is ionic memristors for neuromorphic computing ${ }^{3-5}$, whereas the main obstacle is the undesired current path flowing through neighboring memory cells in the crossbar array architecture, resulting in write/read inaccuracy and unnecessary energy consumption $^{6}$. To overcome this issue, ionic memristors must possess a high current rectification or nonlinearity, which can be solved by artificially connecting a series of transistors ${ }^{7}$, diodes ${ }^{8}$, or nonlinear selectors ${ }^{9}$ for constructing self-nonlinearity/self-rectifying. However, these complex methods inevitably bring sophisticated micro-nano fabrication/integration, low stacking density, and incompatible $3 \mathrm{D}$ vertical crossbar array under certain circumstances. Therefore, developing a single memristor system with self-rectifying characteristics is desirable due to its simplicity, but the progress towards this goal is hampered to a large extent by a lack of a suitable material.

Hitherto, the research of single memristors mainly focuses on oxide-based multicomponent layers by tuning oxygen defects or a single insulating material by grafting the Ag filament. The former excessively depends on a multilayer interface and inevitably suffers from the current leakage from oxygen defects ${ }^{10,11}$. The latter needs assistance by doping fast diffusive silver ions or using active silver electrodes ${ }^{12,13}$. It's also worth mentioning that the current developed ionic memristors usually show a unidirectional rectifying direction. For example, in Ag-based memristors, the relatively abrupt set process makes it difficult to continuously control the conducting states and current rectifying direction as a response to external stimuli ${ }^{14}$. However, these dynamic tunable features are critical for bidirectional operation mode and environment-adaptable learning through the neural network as a response to dynamic environmental stimuli. Therefore, a singlephase memristor simultaneously with highly mobile ions and continuously tunable feature is in urgent need of exploration.

Electric field tuning of physical properties in Van der Waals materials is a very lively field ${ }^{15-17}$. In recent years, the memristor behavior of $2 \mathrm{D}$ van der Waals material has also aroused great attentions ${ }^{18-20}$. One veritable star compound from the thio/selonophosphate family, $\mathrm{CuInP}_{2} \mathrm{~S}_{6}$ (CIPS), meets the requirements for a tunable single-phase memristor from the following three advantages. (1) An identifying feature of this material is the electrochemically active $\mathrm{Cu}^{+}$ions in lattice, which endows itself with an excellent insulating property at a low voltage, and exhibits highly sensitive response of current to electric stimuli above a threshold voltage $^{18,21}$. (2) Due to the van der Waals interaction, CIPS can be easily exfoliated and transferred to desired substrates. (3) Current developed ionic memristors are mostly based on metallic oxide materials. These materials are usually rigid and non-piezoelectric, which are insensitive to external mechanical stimuli. On the contrary, CIPS material (Cc phase) is the only discovered 2D van der Waals ferroionic material so $\mathrm{far}^{22}$, and possesses good flexibility and piezoelectric characteristic ${ }^{22,23}$, which makes it sensitive to the external strain/strain gradient field.

Taking above mentioned advantages, in this work, we study the ionic migration of $\mathrm{Cu}^{+}$and current rectifying behavior in singlephase CIPS materials by using conductive scanning tips to apply different bias and mechanical stimuli. A 120-nm-thick CIPS single material shows a high ON/OFF ratio of $\sim 10^{3}$ along with a self-rectifying ratio of $\sim 10^{3}$. More importantly, this material possesses an excellently continuous modulation of current and rectifying direction by different bias (including the amplitude, speed, and direction) and mechanical strain, which is physically based on the synergetic contribution of controllable $\mathrm{Cu}^{+}$ions migration and interfacial Schottky barrier. These outstanding sensitive features make this material possible for being superior candidate for smart memristors with bidirectional operation mode and strong recognition to input faults and variations.

\section{Results}

The crystal structure of $\mathrm{CuInP}_{2} \mathrm{~S}_{6}$ can be described as a sulfur framework in which metal cations $(\mathrm{Cu}$ and $\mathrm{In})$ and $\mathrm{P}-\mathrm{P}$ pairs fulfill the octahedral voids, as shown in Fig. 1a. In this system, the charged $\mathrm{Cu}^{+}$ions become mobile across the lattice periodic potentials under a high electric field, which is the fundamental of ionic conductivity ${ }^{24}$. The as-grown CIPS single crystal shows a natural $a b$-plane. The sample quality is characterized by performing XRD and SEM-EDS experiments, as shown in Fig. $1 \mathrm{~b}$ and Supplementary Fig. 1, respectively. For the electrical measurements, CIPS nanoflakes were mechanically exfoliated and subsequently taped on conductive Au electrode (see the details in Methods Section). The schematic experimental setup is shown in Fig. 1c, the conductive scanning tip is utilized as the top nanoelectrode, which facilitates the manipulation of a localized conductive path ${ }^{14,25}$. The voltage was applied to the bottom $\mathrm{Au}$ electrode and all the electrical measurements in our experiments were conducted on 120-nmthick CIPS nanoflakes, see Supplementary Fig. 2.

Given the slow mobility of ions, ions migration can be more reliably controlled by the sweeping speed of electric field. Continuous modulation of current by changing the voltage sweeping speed was demonstrated. A series of semi-cycle of $I-V$ curves on two different ferroelectric domains (Fig. 1d) were consecutively measured by gradually reducing the sweeping speed from $12.0 \mathrm{~V} /$ $\mathrm{s}$ to $1.2 \mathrm{~V} / \mathrm{s}$, and the corresponding results are shown in Fig. 1e. When the voltage is swept forward from $0 \mathrm{~V}$ to $3 \mathrm{~V}$ with fast sweeping speed $(>4.8 \mathrm{~V} / \mathrm{s})$, CIPS is in a high insulating state, so called current-off state. As the sweeping speed decreases below $4.8 \mathrm{~V} / \mathrm{s}$, the current off-on transition occurs at a certain threshold voltage and the current increases sharply with a lower sweeping speed, which produces a clear current hysteresis as the voltage is swept backward from $3 \mathrm{~V}$ to $0 \mathrm{~V}$. The higher current at a slower sweeping speed (conduction activation) is a fingerprint of ionic conductors/memristors ${ }^{18}$, as a slow sweeping speed allows $\mathrm{Cu}^{+}$ ions to migrate sufficiently. The cross-sectional SEM-EDS measurement further supports the electrical field-driven $\mathrm{Cu}^{+}$immigration, as shown in Supplementary Fig. 3. Note that the $I-V$ results on two different domains show no obvious difference, ruling out the effect of the ferroelectric polarization and further highlighting the dominant role of the mobile $\mathrm{Cu}^{+}$ions in such sweeping speed-controlled current and current hysteresis. When the voltage is swept from $0 \mathrm{~V}$ to $5 \mathrm{~V}$ at the speed of $2.0 \mathrm{~V} / \mathrm{s}$, the current-off state is switched to current-on state at a threshold voltage of $\sim 3.8 \mathrm{~V}$ due to the activated $\mathrm{Cu}^{+}$ions migration, which contributes to a high ON-OFF ratio of $\sim 10^{3}$ at $2.5 \mathrm{~V}$ as the voltage is swept backward from $5 \mathrm{~V}$ to $0 \mathrm{~V}$. When the voltage is swept from $0 \mathrm{~V}$ to $-5 \mathrm{~V}$, almost no current flows is detected due to the rectifying function of the interfacial Schottky barrier, which contributes to a self-rectifying ratio $\left(\mathrm{I}_{+2.5 \mathrm{~V}} / \mathrm{I}_{-2.5 \mathrm{~V}}\right)$ of $\sim 10^{3}$ at $2.5 \mathrm{~V}$ (Fig. 1f). Note that a negative voltage reconfigures the distribution of $\mathrm{Cu}^{+}$ions and resets the high conducting state into the insulating state again, leading to the repeatable full-cycle of $I-V$ curves as shown in Supplementary Fig. 4.

The sweeping speed-modulated current rectification was further investigated with different sweeping frequencies and opposite voltage directions. As shown in Fig. $2 \mathrm{a}$, in the semi-cycle with a positive voltage, a slow sweeping speed, i.e. $2.4 \mathrm{~V} / \mathrm{s}$, is required to initiate the $\mathrm{Cu}^{+}$ions migration accompanied by the current boost and hysteresis, which can be further enhanced by a slower sweeping speed of $1.2 \mathrm{~V} / \mathrm{s}$. However, the current shows a strikingly different response in the semi-cycle with negative voltage, as shown in Fig. 2b. It is clearly observed that the current boosts at a 

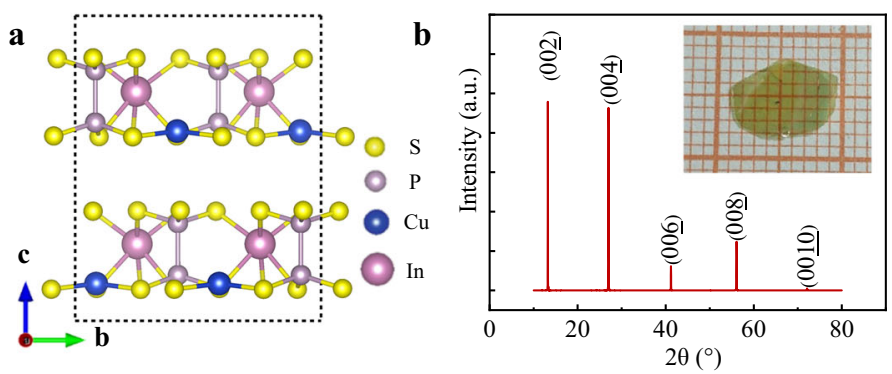

d

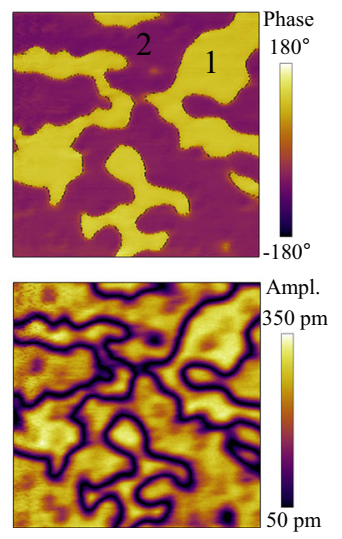

e

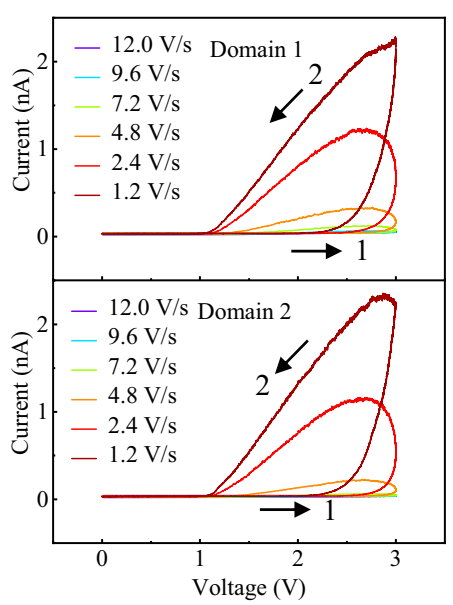

c

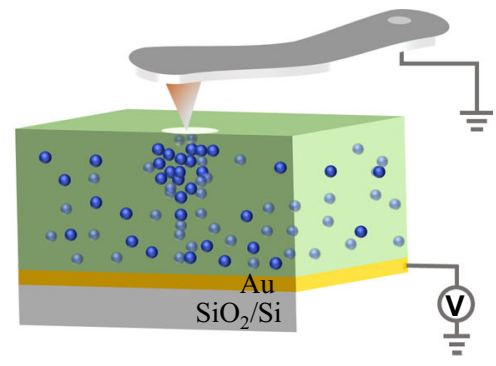

f

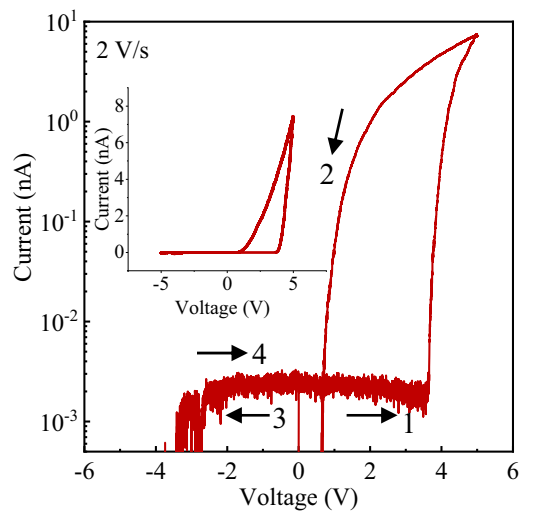

Fig. 1 Characterizations of CIPS single crystal and its electrical properties. a Culn $\mathrm{P}_{2} \mathrm{~S}_{6}$ crystal structure with a vdW gap between the layers; $\mathbf{b}$ The measured XRD pattern for as-grown CIPS single crystal with ab crystalline plane, the inset shows the flexible CIPS crystal. c The schematic experimental setup for electrical measurement. $\mathrm{Cu}^{+}$ions are represented by the blue balls, which undergoes directional migration driven by the external electric field. $\mathbf{d}$ Out of plane PFM phase and amplitude images of CIPS flakes, respectively; e The consecutive sweeping speed-dependent $I-V$ semi-cycles $(0 \mathrm{~V} \rightarrow+3 \mathrm{~V} \rightarrow 0 \mathrm{~V})$ measured on two different domains with opposite direction in d. f A full cycle of $I-V$ curve by sinusoidal voltage sweeping of $0 \mathrm{~V} \rightarrow 5 \mathrm{~V} \rightarrow-5 \mathrm{~V} \rightarrow 0 \mathrm{~V}$ at a sweeping speed of $2 \mathrm{~V} / \mathrm{s}$. The thickness of CIPS is $120 \mathrm{~nm}$.

sweeping speed of $12.0 \mathrm{~V} / \mathrm{s}$, continuously increases until reaching a "peak value" at the speed of $4.8 \mathrm{~V} / \mathrm{s}$, and then starts a dramatic decline. The current hysteresis also shows changes in response to a slower sweeping speed, with its gap continuously reducing and almost disappearing at the sweeping speed of $4.8 \mathrm{~V} / \mathrm{s}$, and then being reversed in the direction. The different current response to the sweeping speed in opposite voltage direction makes it possible for the sweeping speed-controlled current rectification. As shown in Fig. 2c, a clear sweeping speed-modulated current rectification is observed, more specifically, the preferential current flow in negative voltage is inversed to positive bias direction as the voltage is swept from $12.0 \mathrm{~V} / \mathrm{s}$ to $1.2 \mathrm{~V} / \mathrm{s}$. The full-cycle of $I-V$ curves with opposite voltage sweeping are also measured and reproduce a similar result (Supplementary Fig. 5). In addition, we conducted a similar electrical measurement using the top macroscopic platinum electrode $\left(1 \times 1 \mu \mathrm{m}^{2}\right)$, as shown in Supplementary Fig. 6, which reproduces the control of current rectifying behavior and further rules out the possible dominating effect of electrochemical reaction with atmosphere in the c-AFM measurement. The $I-V$ curves with the consecutively forward $(-3 \mathrm{~V} \rightarrow+3 \mathrm{~V})$ and backward sweeping $(+3 \mathrm{~V} \rightarrow-3 \mathrm{~V})$ also reveal the function of self-rectifying (Fig. 2d), i.e, with $6.0 \mathrm{~V} / \mathrm{s}$ sweeping speed, the forward and backward sweeping induces the current flow in the reversed direction. In addition to the sweeping speed-controlled self-rectifying, the magnitude of sweeping voltage and sweeping cycles can also tune the self-rectifying, as shown in Supplementary Figs. 7 and 8. Due to the nature of the time-dependent $\mathrm{Cu}^{+}$ immigration, pulse frequency also modulates the current rectifying behavior similar to the sweeping speed, as shown in Supplementary Figs. 9 and 10. As the pulse frequency increases, the current in the positive voltage starts to increase, however, the current in the negative voltage starts to decrease, which reverses the direction of current flow. The sensitivity of current and rectifying behavior to sweeping parameters and pulse frequency manifests its potential application in future neurofunction in response to complex environmental conditions.

In general, current rectifying behavior usually occurs at asymmetric interfaces such as $p-n$ junctions or Schottky barriers, where electrons and holes are the carriers of the entire system ${ }^{26}$. However, in ionically active CIPS materials, both $\mathrm{Cu}^{+}$ions and electron carriers participate in the electrical transport. The slow redistribution process of $\mathrm{Cu}^{+}$ions under the electric field can be reliably controlled by the sweeping speed, which is the key to revealing the underlying mechanism for the sweeping speedcontrolled self-rectifying behavior. From the energy band configuration, as sketched in Fig. 3a, the difference in the work function/electron affinity creates asymmetric interfaces, with the Schottky barrier $\varphi_{1}$ and $\varphi_{2}$ at the side of Pt/Ir tip and the bottom $\mathrm{Au}$ electrode, respectively. In the absence of $\mathrm{Cu}^{+}$ions migration, as shown in Fig. 3b, the electrons need to overcome a larger barrier $\varphi_{1}$ under positive voltage and a smaller barrier $\varphi_{2}$ under negative voltage for current flow, which leads to smaller voltage drops across the CIPS under positive voltage. In the presence of $\mathrm{Cu}^{+}$migration, the $\mathrm{Cu}^{+}$ions will undergo directional migration driven by the electric field and generate a localized conducting path under the tip, as shown in Fig. 3c, d. The fast/slow sweeping speed corresponds to the case of weak/strong $\mathrm{Cu}^{+}$migration, respectively. In positive voltage sweeping as shown in Fig. 2a, almost no current flow is detected at a sweeping speed faster than $4.8 \mathrm{~V} / \mathrm{s}$ due to the larger interface barrier $\varphi_{1}$ and weak $\mathrm{Cu}^{+}$ 

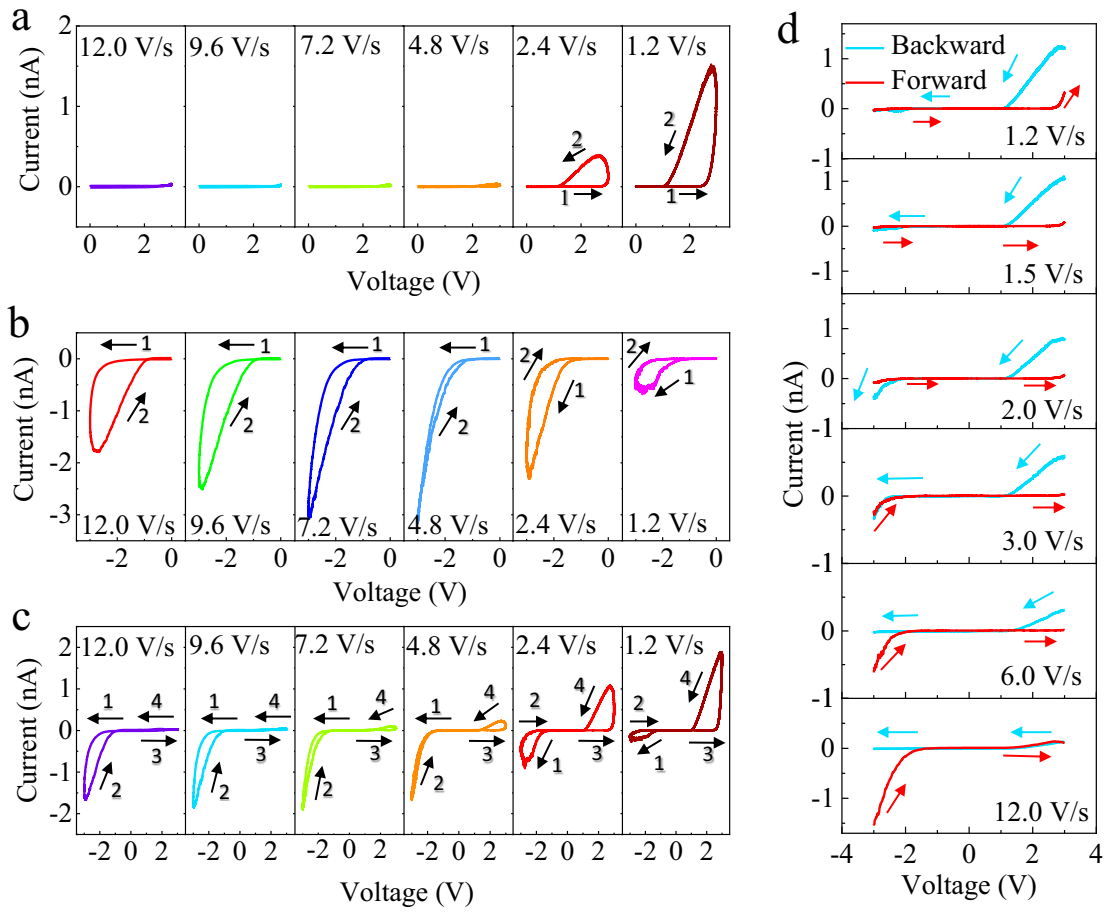

Fig. 2 Current rectification modulated by the sweeping speed/direction. a-c Response of current-voltage (I-V) curves to sweeping speed with a sinusoidal voltage sweeping semi-cycle of $(0 \mathrm{~V} \rightarrow+3 \mathrm{~V} \rightarrow 0 \mathrm{~V}),(0 \mathrm{~V} \rightarrow-3 \mathrm{~V} \rightarrow 0 \mathrm{~V})$, and full-cycle of $(0 \mathrm{~V} \rightarrow-3 \mathrm{~V} \rightarrow+3 \mathrm{~V} \rightarrow 0 \mathrm{~V})$, respectively; $l-V$ curves are consecutively measured at a gradually lower sweeping speed ranging from $12.0 \mathrm{~V} / \mathrm{s}$ to $1.2 \mathrm{~V} / \mathrm{s}$. The numbers $1-4$ denote the sweeping sequence. $\mathbf{d}$ Response of $I-V$ curves measured by the forward voltage sweeping $(-3 \mathrm{~V} \rightarrow+3 \mathrm{~V})$ and backward voltage sweeping $(+3 \mathrm{~V} \rightarrow-3 \mathrm{~V})$ at a different sweeping speeds ranging from $12.0 \mathrm{~V} / \mathrm{s}$ to $1.2 \mathrm{~V} / \mathrm{s}$ on two adjacent sites.

migration. As sweeping speed decreases below $4.8 \mathrm{~V} / \mathrm{s}$, the current increases sharply due to the enhanced $\mathrm{Cu}^{+}$migration. Note that more $\mathrm{Cu}^{+}$ions migrate toward and concentrate around the tip in this process and generate a " $\mathrm{Cu}^{+}$ions-rich" region, which reduces the Schottky barrier $\varphi_{1}$ under the tip via an ionic capacitive effect $^{27,28}$ or electrochemistry reaction ${ }^{29}$, as shown in Fig. 3e. The reduction of Schottky barrier $\varphi_{1}$ further synergistically boosts the current by distributing more voltage drops on CIPS to enhance the $\mathrm{Cu}^{+}$ions migration. In negative voltage sweeping as shown in Fig. $2 \mathrm{~b}$, although at a fast sweeping speed of $12.0 \mathrm{~V} / \mathrm{s}, \mathrm{Cu}^{+}$ions migration can be easily initiated due to the lower interface barrier $\varphi_{2}$, which boosts the current. However, as shown in Fig. $3 \mathrm{f}, \mathrm{Cu}^{+}$ ions are also expelled from the tip in negative voltage sweeping to generate $\mathrm{a}^{\mathrm{C}} \mathrm{Cu}^{+}$ions-deficient" region under the tip and imposes a higher potential barrier $\varphi_{1}$. In this case, the competition between $\mathrm{Cu}^{+}$ions migration and the interfacial Schottky barrier change exists in negative voltage sweeping, which depends on the sweeping speed. As the sweeping speed decreases below $4.8 \mathrm{~V} / \mathrm{s}$, it is difficult to continuously drive $\mathrm{Cu}^{+}$ion migration in the bulk, leading to the dramatic decline in current accompanied by a reversal of hysteresis, as shown in Fig. 2b. The migration of $\mathrm{Cu}^{+}$ ions can be also verified from the topographic changes ${ }^{30}$, as shown in Fig. 3g-i, where a slightly topographic bump ( $\sim \mathrm{nm})$ appears with $I-V$ measurement in the white square area after positive voltage sweeping, and can be reversibly eliminated by the subsequent negative voltage sweeping.

In addition to the sweeping speed-controlled current rectifying, the mechanical modulation of current rectifying is also revealed. As a simple demonstration, the local strain/strain gradient is applied by the scanning tip. With a small tip force of $0.35 \mu \mathrm{N}$ as the contact force, the $I-V$ curve shows unidirectional current flow in positive voltage direction, as shown in Fig. 4a. As the tip force is gradually increased to $4.20 \mu \mathrm{N}$ (Fig. 4b), the current under positive voltage gradually increases and eventually reaches its saturation value above $1.40 \mu \mathrm{N}$, while the current under negative voltage increases rapidly and even exhibits a larger value than that under positive bias above $2.80 \mu \mathrm{N}$. The current dependence on tip force in ON state at $\pm 5 \mathrm{~V}$ is shown in Fig. 4c. The bidirectional current-on state can be repeatedly reset to current-off state by a reverse voltage, which resembles a switchable current rectifying. The modified thermionic emission theory is further used to describe the interfacial electron injection in the case of $\mathrm{Cu}^{+}$ions migration (see the details in Supplementary Figs. 11 and 12). Here we introduce a $\beta$ factor to describe the influence of the $\mathrm{Cu}^{+}$ions migration and the modified $\mathrm{I}-\mathrm{V}$ rectifying behavior. The value of $\beta=0,<0.5$ and $>0.5$ represents the case of no $\mathrm{Cu}^{+}$ions migration, a stronger $\mathrm{Cu}^{+}$ions migration in negative voltage direction, and a stronger $\mathrm{Cu}^{+}$ions migration under positive bias, respectively. $\Delta \varphi_{1}$ represents the Schottky barrier change under the mechanical load. The I-V data in bidirectional current-on state in Fig. $4 \mathrm{~b}$ was simulated with modified thermionic emission theory. As shown in Fig. $4 \mathrm{~d}-\mathrm{f}$, as the tip force increases from $0.35 \mu \mathrm{N}$ to $4.20 \mu \mathrm{N}$, $\varphi_{1}$ decreases with a value of $\Delta \varphi_{1}=0.47 \mathrm{eV}$, which synergistically enhances the $\mathrm{Cu}^{+}$ions migration under negative voltage by distributing more voltages drops on CIPS. This phenomenon is manifested by the $\beta$ factor decreasing from 0.83 to 0.38 . Note that tip force-induced bandgap or contact area change is not the dominating factor, as shown in Supplementary Figs. 13 and 14.

Herein, the Schottky barrier change is probably related to strain and strain gradient under the AFM tip ${ }^{23,31}$, which induces an additional electric field from the piezoelectric effect and flexoelectric effect (Fig. 4g, i) and therefore synergetically reduces the Schottky barrier under the tip. The calculated strain and strain gradient are shown in Supplementary Fig. 15. The induced surface potentials from these two effects are shown in Supplementary Fig. 16. The effective surface potentials for piezoelectric and flexoelectric effect near the tip are $1.06 \mathrm{~V}$ and $-0.56 \mathrm{~V}$, respectively, and together give a net surface 

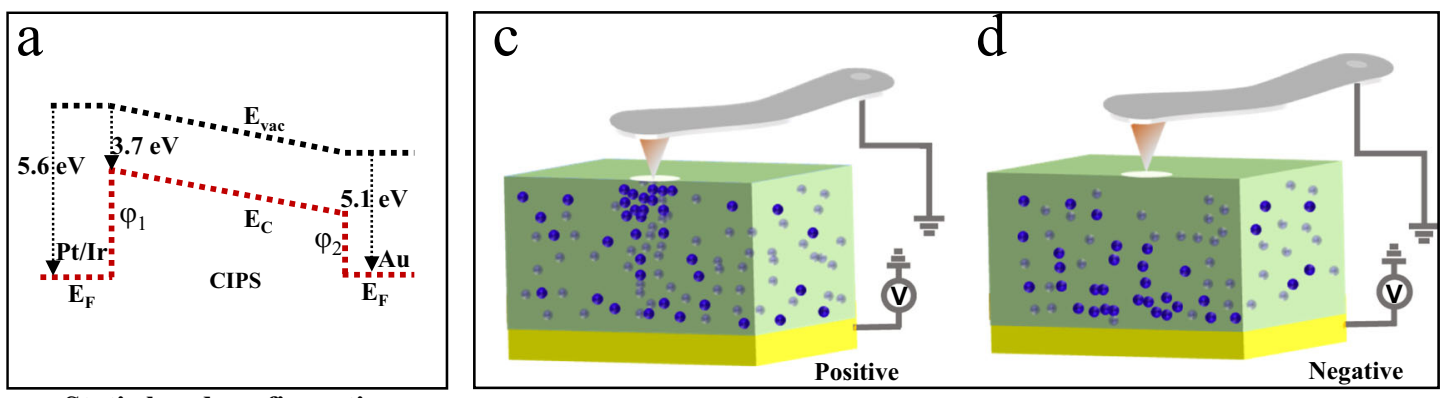

Static band configuration
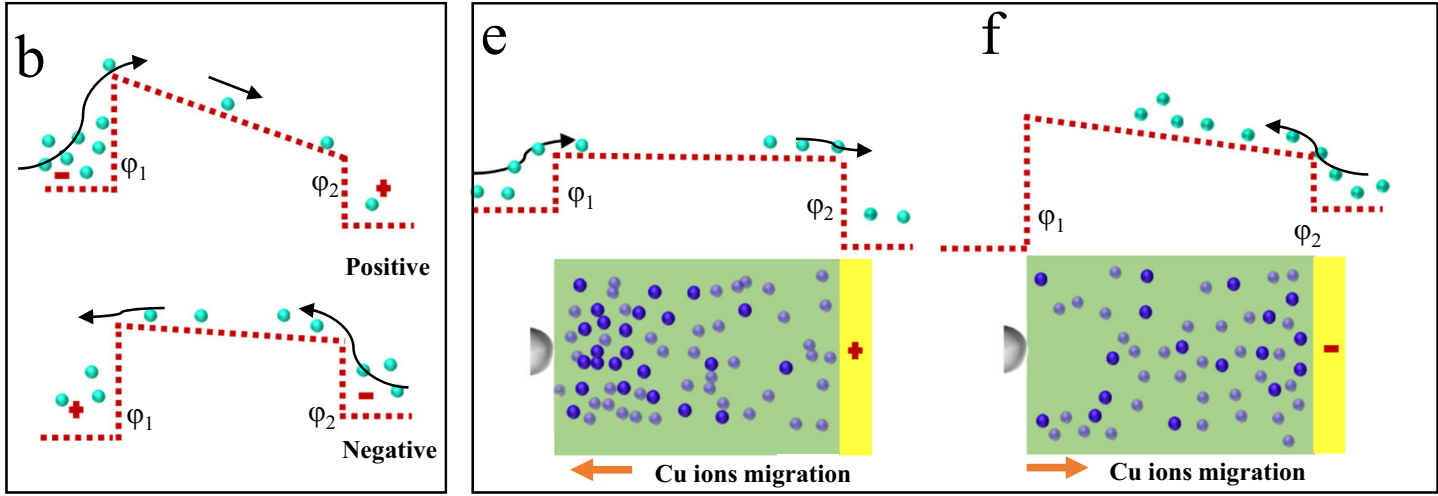

No $\mathrm{Cu}$ ions migration

$\mathrm{Cu}$ ions migration
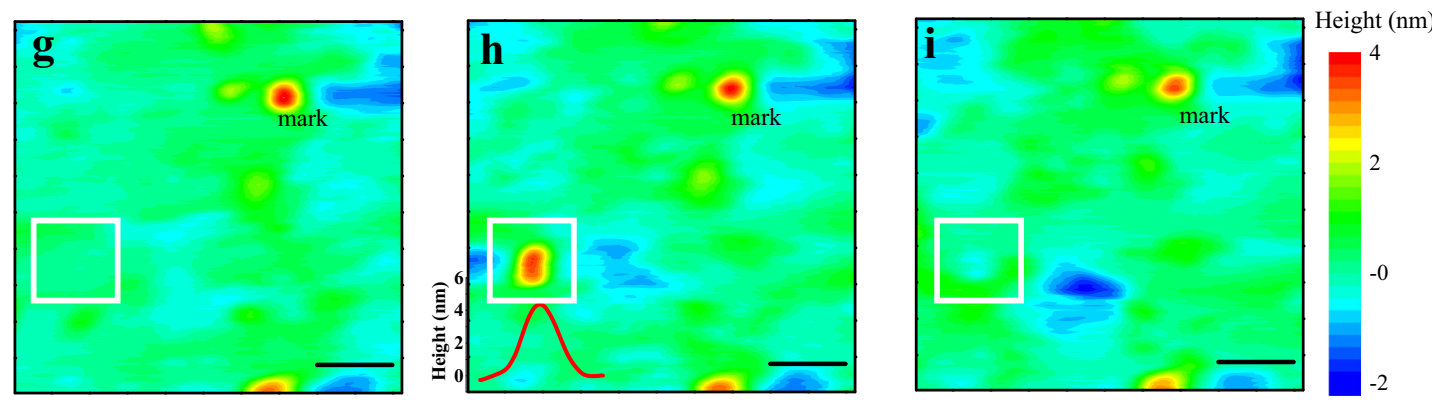

Fig. 3 Physical mechanism of highly mobile $\mathbf{C u}^{+}$ions-meditated current rectification. a Schematic static band configuration for tip/CIPS/Au bottom electrode measurement setup according to the work function of Pt/Ir tip $(5.6 \mathrm{eV})^{42}$, Au $(5.1 \mathrm{eV})^{43}$, and electron affinity of CIPS (3.7 eV) 44 . The difference in the work function/electron affinity creates asymmetric interfaces, with the Schottky barrier $\varphi_{1}$ and $\varphi_{2}$ at the side of Pt/Ir tip and the bottom Au electrode, respectively; $\mathbf{b}$ In the absence of $\mathrm{Cu}^{+}$ions migration, the schematic electrons (cyan balls) transport process in different bias direction, where the bias voltage is applied to the bottom Au substrate; $\mathbf{c}, \mathbf{d} \mathbf{C u}^{+}$ions (blue ball) undergo directional migration driven by the applied voltage. $\mathbf{e}, \mathbf{f} \mathbf{I n}$ the presence of $\mathrm{Cu}^{+}$ions migration and redistribution, the schematic $\mathrm{Cu}^{+}$ions (blue balls) migration and electrons (cyan balls) transfer for current flow. $\mathbf{g}-\mathbf{i}$ The topography image in virgin state, after two consecutive positive voltage sweeping semi-cycle $(0 \mathrm{~V} \rightarrow+4 \mathrm{~V} \rightarrow 0 \mathrm{~V})$, and after two consecutive negative voltage sweeping semi-cycle $(0 \mathrm{~V} \rightarrow-4 \mathrm{~V} \rightarrow 0 \mathrm{~V})$, respectively. The $I-V$ measurements are conducted in the white square area in $\mathbf{g}$-i and the ever-present bump in the mark location rules out the possibility of tip scraping for the bump elimination during scanning. The scale bar length is $200 \mathrm{~nm}$ in $\mathbf{g}$-i.

potential of $\sim 0.5 \mathrm{~V}$, which is close to the Schottky barrier change of $\sim 0.47 \mathrm{~V}$ under the tip force of $4.20 \mu \mathrm{N}$. Finally, we conducted the consecutive writing $( \pm 5 \mathrm{~V})$ and reading $( \pm 3 \mathrm{~V})$ bias pulse measurement at a tip force of $4.20 \mu \mathrm{N}$, as shown in Fig. 4i. The bidirectional threshold memory with self-rectifying function is manifested in the ability to control the current flow, where a large positive/negative voltage pulse in writing operation leads to the positive/negative rectification $(\mathrm{PR} / \mathrm{NR})$ in the small voltage reading operation. This dynamic characteristic of bidirectional threshold memory is critical for bidirectional operation mode and environment-adaptable learning through the neural network as a response to dynamic environmental stimuli.

\section{Discussion}

In summary, we report a single-phase memristor based on van der Waals material, $\mathrm{CuInP}_{2} \mathrm{~S}_{6}$ (CIPS), which satisfies continuously tunable electric features. An identifying feature of this material is the electrochemically active $\mathrm{Cu}^{+}$ions, which endows CIPS with a superior continuous modulation of current and self-rectifying by the sweeping speed of voltage, voltage direction, voltage amplitude, etc. or mechanical strain. The synergetic contribution of controllable $\mathrm{Cu}^{+}$ions migration and interfacial Schottky barrier change is proposed to control the current flow in the bulk as well as the injection-limited electrode contact. The dynamically tunable feature is in urgent demand for further evolution of smart memristors with bidirectional operation mode and strong recognition to input faults and variations.

\section{Methods}

Sample preparation. CIPS Single crystals were synthesized by the chemical vapor transport method using stoichiometric elemental precursors ${ }^{32,33}$. The precursors were sealed inside a vacuumed quartz ampoule and then subjected to a two-zone horizontal 
a

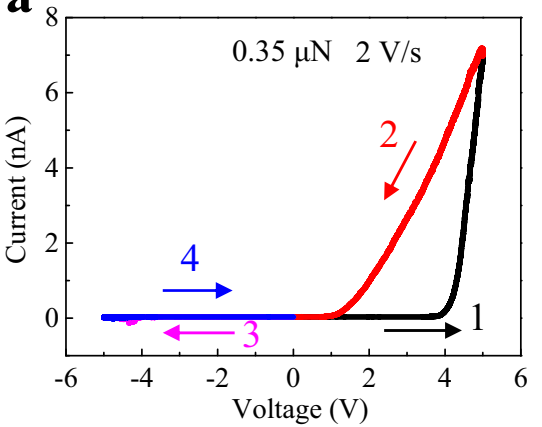

d
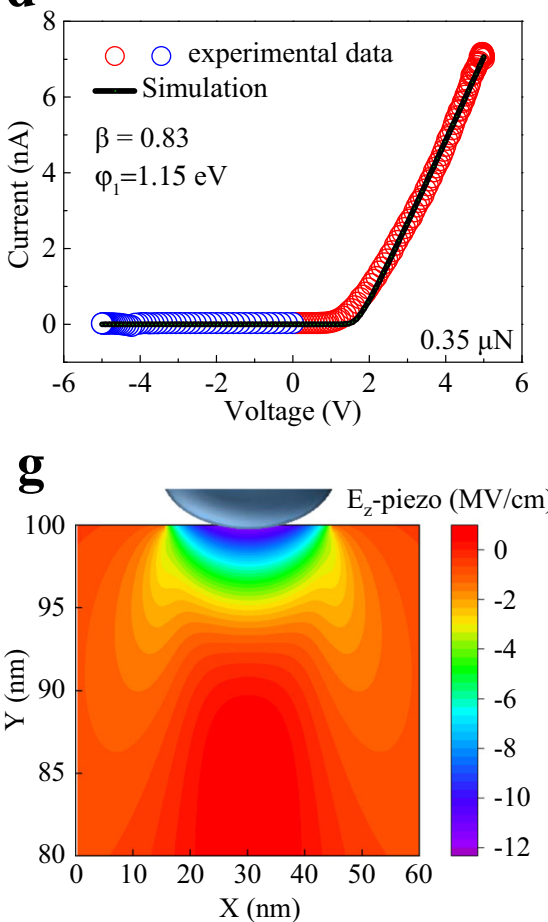

b

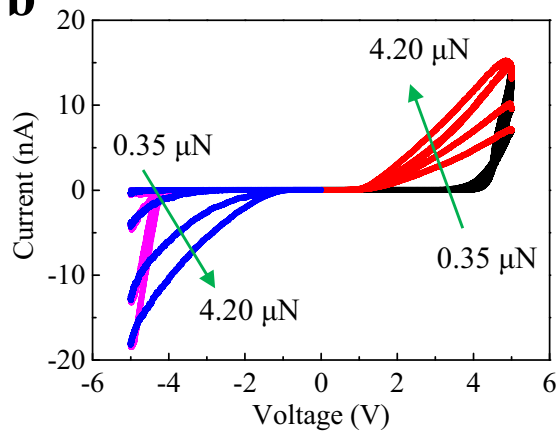

e
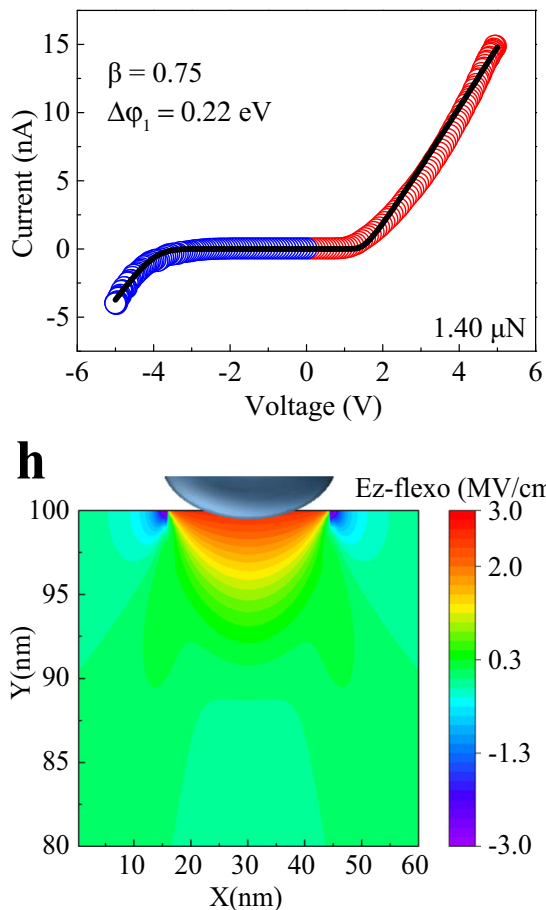

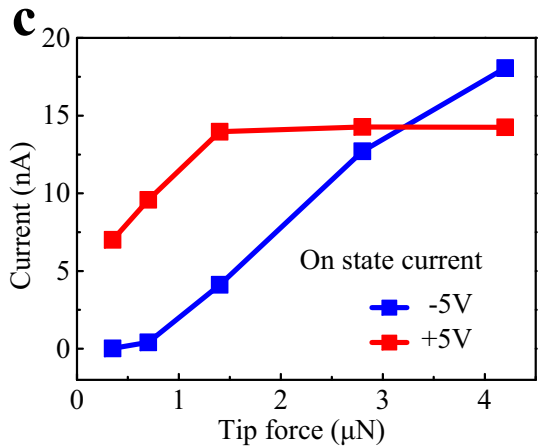

f

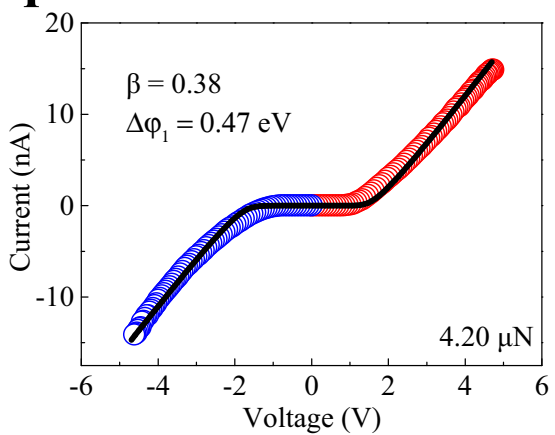

\section{i}

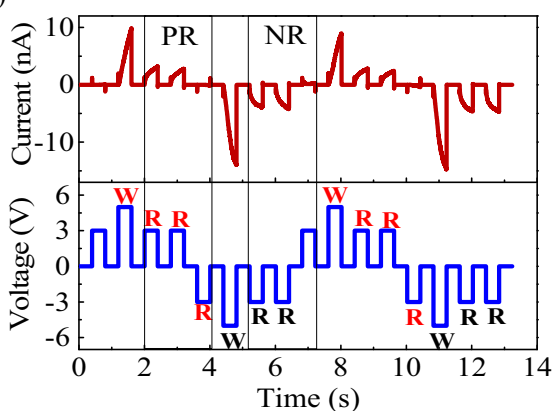

Fig. 4 The mechanical modulation of current rectifying in CIPS via the AFM tip force. a The $I-V$ curves with a voltage sweeping of $0 \mathrm{~V} \rightarrow+5 \mathrm{~V} \rightarrow-5 \mathrm{~V} \rightarrow 0 \mathrm{~V}$ measured at a sweeping speed of $2 \mathrm{~V} / \mathrm{s}$. The number $1-4$ denotes the sweeping sequence in a full cycle. The initial tip compressive force of $0.35 \mu \mathrm{N}$ is set to the contact force between tip and nanoflakes. $\mathbf{b}$ The $I-V$ curves with a voltage sweeping ( $0 \mathrm{~V} \rightarrow+5 \mathrm{~V} \rightarrow-5 \mathrm{~V} \rightarrow 0 \mathrm{~V}$ ) at a sweeping speed of $2 \mathrm{~V} / \mathrm{s}$, but with different tip compressive force: $0.35 \mu \mathrm{N}, 0.70 \mu \mathrm{N}, 1.40 \mu \mathrm{N}, 2.80 \mu \mathrm{N}, 4.20 \mu \mathrm{N}$. c The current in ON state at $\pm 5 \mathrm{~V}$ versus tip force. $\mathbf{d}-\mathbf{f}$ The $I-V$ data in ON state in positive (red) and negative voltage (blue) at different tip force is simulated (black line) by the modified thermionic emission theory; $\mathbf{g}, \mathbf{h}$ the piezoelectric field ( $E_{z}$-piezo) and flexoelectric field ( $\left.E_{z}-f l e x o\right)$ in CIPS flakes obtained from theoretical calculations with a tip-force model under a tip force of $4.20 \mu \mathrm{N}$, respectively. i A consecutive write ( $\pm 5 \mathrm{~V})$ and read $( \pm 3 \mathrm{~V})$ voltage pulses measurement at a constant tip force of $4.20 \mu \mathrm{N}$. A large positive/negative voltage pulse in writing operation leads to the positive/negative rectification (PR/NR) in the small voltage reading operation.

tube furnace for a reaction time of 212 hours, the hot zone and cold zone were set to 650 and $600{ }^{\circ} \mathrm{C}$, respectively. The thin flakes were obtained by mechanical exfoliation, where the as-grown single crystal was first attached to the adhesive tape, then repeatedly folded and tore off, and finally transferred to the conductive $\mathrm{Au}(5 \mathrm{~nm}) /$ $\mathrm{SiO}_{2}(285 \mathrm{~nm}) / \mathrm{Si}$ substrate. Au was deposited by electron-beam evaporation in high vacuum of $\sim 10^{-6} \mathrm{mbar}$. Macroscopic top platinum electrode was also deposited by electron beam using GIS (Gas Injection System) attachment in FIB (Focused Ion beam) system. The voltage and current parameters for electron beam deposition was $10 \mathrm{kV}$ and $1.7 \mathrm{nA}$, respectively. For the characterization of $\mathrm{Cu}+$ immigration under the electric field, bulk CIPS ( $15 \mu \mathrm{m}$ thickness) sandwiched between two gold electrodes was prepared and the cross-sectional areas are exposed for the SEM-EDS measurement immediately after removing the bias.

Scanning probe microscopy measurement. Vector PFM and c-AFM measurements were performed using a commercial atomic force microscope (Asylum Research MFP-3D) with Pt/Ir-coated Si cantilever tips with a radius of $\sim 25 \mathrm{~nm}$ and spring constants of $\sim 0.5-9.5 \mathrm{~N} / \mathrm{m}$. In vector PFM mode, ferroelectric domains were imaged by the tip driven with an ac voltage $\left(V_{\mathrm{ac}}=0.5-1 \mathrm{~V}\right)$. The PFM was acquired at the drive frequency of $\approx 350 \mathrm{kHz}$ in Vector PFM mode. In c-AFM measurement, single point current-voltage $(I-V)$ measurements were performed with ORCA module with compliance current of $20 \mathrm{nA}$. The voltage was applied to the conducting Au bottom electrode, which was continuously swept to simultaneously read the current. The precise tip force was calculated by multiplying the spring constant, the inverse optical lever sensitivity (InvOLS), and setpoint. All these $I-V$ measurements were performed at ambient temperature.

\section{Simulation and calculation}

$\mathrm{I}-\mathrm{V}$ simulation. The electrical measurement setup was described by a circuit model for the AFM tip/CIPS flake/Au substrate. The tip-flake and flake-substrate interfaces were represented as Schottky barriers connected in a back-to-back geometry. A resistance, R, connected in series between the two Schottky barriers accounts for bulk resistance of the CIPS flake. The modified thermionic emission theory is described by ${ }^{34}$ :

$$
\begin{gathered}
I(V)=I_{0} \exp ^{\left(-\beta V / k_{B} T\right)}\left(\exp ^{\left(q V / k_{B} T\right)}-1\right) \\
I_{0}=A_{c} A^{*} T^{2} \exp ^{\left(-q \varphi_{b} / k_{B} T\right)}
\end{gathered}
$$

where $I_{0}$ is the saturation current of the junction, $q$ is the electron charge, $V$ is the 
applied voltage, $k_{\mathrm{B}}$ is the Boltzmann constant, $T$ is the temperature, $A_{\mathrm{c}}$ is the Schottky barrier contact area, $A^{*}$ is the effective Richardson constant, and $\varphi_{\mathrm{b}}$ is the initial energy of the Schottky barrier, $\beta$ factor is introduced to mimic the influence of mobile $\mathrm{Cu}^{+}$ion on the profiles of $I-V$ curves and mainly describes the degree of $\mathrm{Cu}^{+}$ions migration in different voltage direction.

Strain and strain gradient calculation. The strain and strain gradient distributions in CIPS flakes were obtained from theoretical simulations with a tip-force model under a tip force of $4.2 \mu \mathrm{N}^{31}$. The Pt/Ir-coated silicon tip was considered as the rigid sphere, the Young's modulus and Poisson's ratio of CIPS are $25 \mathrm{GPa}$ and -0.06 , respectively ${ }^{23,35}$. The piezoelectric and flexoelectric fields were obtained by using the linear piezoelectric equation and the flexoelectric constitutive equation, respectively. The surface potentials induced by piezoelectric and flexoelectric effects were obtained by integrating the piezoelectric field and flexoelectric field. The details of calculations can be found in supplementary materials.

Density functional theory calculation. In this work, the effect of vertical strain on bandgap of CIPS was investigated using the Vienna Ab initio Simulation Package (VASP) ${ }^{36,37}$ based on the density functional theory (DFT). The electron-ion interactions were described by Projector-augmented-wave (PAW) potentials ${ }^{38}$, while Perdew-Burke-Ernzerhof functional of generalized gradient approximation ${ }^{39}$ was chosen to deal with the exchange-correlation functional. The cut-off energy of $450 \mathrm{eV}$ was for the plane-wave basis and the Monkhorst-Pack mesh ${ }^{40}$ with $\mathrm{k}$-spacing of $0.2 \AA^{-1}$ was set for k-point sampling. And the energy and force convergence criterions were $10^{-6} \mathrm{eV}$ and $10^{-3} \mathrm{eV} / \AA^{-1}$, respectively. In order to take into account the interlayer van der Waals interaction in bulk CIPS, DFT-D2 method $^{41}$ was adopted.

\section{Data availability}

The data that support the findings of this study are available from the corresponding author on request.

Received: 27 August 2021; Accepted: 13 January 2022;

Published online: 31 January 2022

\section{References}

1. Kalinin, S. V. \& Spaldin, N. A. Functional ion defects in transition metal oxides. Science 341, 858-859 (2013).

2. Yang, Y. \& Huang, R. Probing memristive switching in nanoionic devices. Nat. Electron. 1, 274-287 (2018).

3. Duan, Q. et al. Spiking neurons with spatiotemporal dynamics and gain modulation for monolithically integrated memristive neural networks. Nat. Commun. 11, 3399 (2020).

4. Wang, Z. et al. Fully memristive neural networks for pattern classification with unsupervised learning. Nat. Electron. 1, 137-145 (2018).

5. Wang, Z. et al. Resistive switching materials for information processing. Nat. Rev. Mater. 5, 173-195 (2020).

6. Linn, E., Rosezin, R., Kügeler, C. \& Waser, R. Complementary resistive switches for passive nanocrossbar memories. Nat. Mater. 9, 403-406 (2010).

7. Yao, P. et al. Fully hardware-implemented memristor convolutional neural network. Nature 577, 641-646 (2020).

8. Kim, G. et al. Resistive memory: $32 \times 32$ crossbar array resistive memory composed of a stacked schottky diode and unipolar resistive memory. Adv. Funct. Mater. 23, 1440-1449 (2013).

9. Midya, R. et al. Anatomy of Ag/Hafnia-based selectors with $10^{10}$ nonlinearity. Adv. Mater. 29, 1604457 (2017).

10. Seok, J. Y. et al. A review of three-dimensional resistive switching cross-bar array memories from the integration and materials property points of view. Adv. Funct. Mater. 24, 5316-5339 (2014).

11. Shi, L. E. A. Research progress on solutions to the sneak path issue in memristor crossbar arrays. Nanoscale Adv. 2, 1811-1827 (2020).

12. Yao, Z. et al. Simultaneous implementation of resistive switching and rectifying effects in a metal-organic framework with switched hydrogen bond pathway. Sci. Adv. 5, w4515 (2019).

13. Waser, R., Dittmann, R., Staikov, G. \& Szot, K. Redox-based resistive switching memories-nanoionic mechanisms, prospects, and challenges. Adv. Mater. 21, 2632-2663 (2009).

14. Berco, D. et al. Nanoscale conductive filament with alternating rectification as an artificial synapse building block. ACS Nano 12, 5946-5955 (2018).

15. Bafekry, A. et al. Graphene hetero-multilayer on layered platinum mineral jacutingaite $\left(\mathrm{Pt}_{2} \mathrm{HgSe}_{3}\right)$ : van der Waals heterostructures with novel optoelectronic and thermoelectric performances. J. Mater. Chem. A. 8, 13248-13260 (2020)
16. Bafekry, A., Stampfl, C. \& Ghergherehchi, M. Strain, electric-field and functionalization induced widely tunable electronic properties in $\mathrm{MoS}_{2} / \mathrm{BC}_{3}, / \mathrm{C}_{3} \mathrm{~N}$ and $/ \mathrm{C}_{3} \mathrm{~N}_{4}$ van der Waals heterostructures. Nanotechnology 31, 295202 (2020).

17. Bafekry, A. \& Neek-Amal, M. Tuning the electronic properties of graphene-graphitic carbon nitride heterostructures and heterojunctions by using an electric field. Phys. Rev. B. 101, 085417 (2020).

18. Zhang, D., Luo, Z. D., Yao, Y., Schoenherr, P. \& Seidel, J. Anisotropic ion migration and electronic conduction in van der Waals ferroelectric CuInP $2 \mathrm{~S}_{6}$. Nano Lett. 2, 995-1002 (2021).

19. $\mathrm{Li}, \mathrm{B}$. C. et al. An electronic synapse based on $2 \mathrm{D}$ ferroelectric $\mathrm{CuInP}_{2} \mathrm{~S}_{6}$. $A d v$. Electron. Mater. 6, 2000760 (2020).

20. Zhu, X. et al. Ionic modulation and ionic coupling effects in $\mathrm{MoS}_{2}$ devices for neuromorphic computing. Nat. Mater. 18, 141-148 (2019).

21. Xu, D., Ma, R., Zhao, Y. F., Guan, Z. \& Duan, C. G. Unconventional out-ofplane domain inversion via in-plane ionic migration in a van der Waals ferroelectric. J. Mater. Chem. C 8, 6966-6971 (2020).

22. Deng, J., et al. Thickness-dependent in-plane polarization and structural phase transition in van der Waals Ferroelectric CuInP $2 \mathrm{~S}_{6}$. Small. 16, e1904529 (2020).

23. You, L. et al. Origin of giant negative piezoelectricity in a layered van der Waals ferroelectric. Sci. Adv. 5, v3780 (2019).

24. Neumayer, S. M. et al. Alignment of polarization against an electric field in van der Waals ferroelectrics. Phys. Rev. Appl. 13, 64063 (2020).

25. Chen, Q. et al. Controlled construction of atomic point contact with 16 quantized conductance states in oxide resistive switching memory. ACS Appl. Electron. Mater. 1, 789-798 (2019).

26. Choi et al. Switchable ferroelectric diode and photovoltaic effect in $\mathrm{BiFeO}_{3}$. Science 5923, 63-66 (2009).

27. Chernyak, L., Jakubowicz, A. \& Cahen, D. Junction electroluminescence from microscopic diode structures in $\mathrm{CuInSe}_{2}$, prepared by electric field-assisted doping. Adv. Mater. 7, 45-48 (1995).

28. Yang, D., Su, X., Li, J., Bai, H. \& Tang, X. Blocking ion migration stabilizes the high thermoelectric performance in $\mathrm{Cu}_{2} \mathrm{Se}$ composites. Adv. Mater. 32, 2003730 (2020).

29. Li, S. et al. Synaptic plasticity and learning behaviours mimicked through $\mathrm{Ag}$ interface movement in an $\mathrm{Ag} /$ conducting polymer/Ta memristive system. J. Mater. Chem. C 1, 5292-5298 (2013).

30. Balke et al. Locally controlled Cu-Ion transport in layered ferroelectric $\mathrm{CuInP}_{2} \mathrm{~S}_{6}$. ACS Appl. Mater. Interfaces 32, 27188-27194 (2018).

31. Wang, L. et al. Flexoelectronics of centrosymmetric semiconductors. Nat. Nanotechnol. 15, 661-667 (2020).

32. Liu, F. et al. Room-temperature ferroelectricity in $\mathrm{CuInP}_{2} \mathrm{~S}_{6}$ ultrathin flakes. Nat. Commun. 7, 12357 (2016)

33. Maisonneuve, V. et al. Room-temperature crystal structure of the layered phase CuIInIIIP $\mathrm{S}_{6}$. J. Alloy. Compd 218, 157-164 (1995).

34. Quereda, J., Palacios, J. J., Agräit, N., Castellanos-Gomez, A. \& RubioBollinger, G. Strain engineering of Schottky barriers in single- and few-layer $\mathrm{MoS}_{2}$ vertical devices. 2D Materials 4, 21006 (2017).

35. Zhang, C., Nie, Y., Aijun, D. U. \& Chemistry, S. O. Intrinsic ultrahigh negative poisson's ratio in two-dimensional ferroelectric $\mathrm{ABP}_{2} \mathrm{X}_{6}$ materials. Acta Phys. Chim. Sin. 32, 1128-1133 (2019).

36. Kresse, G. G. \& Furthmüller, J. J. Efficient iterative schemes for ab initio totalenergy calculations using a plane-wave basis set. Phys. Rev. B Condens. Matter 54, 11169 (1996).

37. Gk, A. \& Jf, B. Efficiency of ab-initio total energy calculations for metals and semiconductors using a plane-wave basis set. Comp. Mater. Sci. 6, 15-50 (1996).

38. Blochl, P. E. Projected augmented-wave method. 50, 17953-17979 (1994).

39. Perdew, J. P. et al. Erratum: Atoms, molecules, solids, and surfaces: applications of the generalized gradient approximation for exchange and correlation. Phys. Rev. B Condens. Matter 46, 6671-6687 (1993).

40. Monkhorst, H. J. \& Pack, J. D. Special points for Brillouin-zone integrations Phys. Rev. B Condens. Matter 13, 5188-5192 (1976).

41. Grimme, S. Semiempirical GGA-type density functional constructed with a long-range dispersion correction. J. Comput. Chem. 27, 1787-1799 (2010).

42. Das, S. et al. Enhanced flexoelectricity at reduced dimensions revealed by mechanically tunable quantum tunnelling. Nat. Commun. 10, 537 (2019).

43. Park, Y. J., Song, A. R., Walker, B., Seo, J. H. \& Chung, K. B. Hybrid ZnON organic light emitting transistors with low threshold voltage $<5 \mathrm{~V}$. Adv. Opt. Mater. 7, 1801290-1801291 (2019).

44. Wu, J., Chen, H. Y., Yang, N., Cao, J. \& Wang, H. High tunnelling electroresistance in a ferroelectric van der Waals heterojunction via giant barrier height modulation. Nat. Electron. 3, 1-7 (2020).

\section{Acknowledgements}

X.Y.W. acknowledges the support by National Key Research and Development Program of China (2019YFA0307900), and the National Natural Science Foundation of China (92163101). J.H. acknowledges the support by National Natural Science Foundation of 
China (12172047). X.L.W. acknowledges the support by National Natural Science Foundation of China (12074018). J.H. and X.Y.W. acknowledge the support by the Beijing Natural Science Foundation (Z190011) and the Beijing Institute of Technology Research Fund Program for Young Scholars.

\section{Author contributions}

X.Y.W., X.L.W., and J.H. designed and supervised the experiments. X.J., J.D., and R.N. prepared the samples. X.J. performed PFM and c-AFM experiments. X.Z. calculated the surface potentials induced by the piezoelectric and flexoelectric effect under the tip. Y.L. performed DFT calculations. XRD experiments were done by S.X. and T.X.; Y.L. provides the help for $I-V$ simulation. All authors discussed the results and commented on the manuscript.

\section{Competing interests}

The authors declare no competing interests.

\section{Additional information}

Supplementary information The online version contains supplementary material available at https://doi.org/10.1038/s41467-022-28235-6.

Correspondence and requests for materials should be addressed to Xueyun Wang, Xiaolei Wang or Jiawang Hong.
Peer review information Nature Communications thanks the other anonymous reviewer(s) for their contribution to the peer review of this work.

Reprints and permission information is available at http://www.nature.com/reprints

Publisher's note Springer Nature remains neutral with regard to jurisdictional claims in published maps and institutional affiliations.

\begin{abstract}
(c) (i) Open Access This article is licensed under a Creative Commons Attribution 4.0 International License, which permits use, sharing, adaptation, distribution and reproduction in any medium or format, as long as you give appropriate credit to the original author(s) and the source, provide a link to the Creative Commons license, and indicate if changes were made. The images or other third party material in this article are included in the article's Creative Commons license, unless indicated otherwise in a credit line to the material. If material is not included in the article's Creative Commons license and your intended use is not permitted by statutory regulation or exceeds the permitted use, you will need to obtain permission directly from the copyright holder. To view a copy of this license, visit http://creativecommons.org/ licenses/by/4.0/.
\end{abstract}

(C) The Author(s) 2022 